\title{
Modeling the Growth of Streamers during Liquid Breakdown
}

\author{
Minkyu Kim, Robert E. Hebner \\ The University of Texas at Austin \\ 1 University Station \#R7000 \\ Austin, TX 78712, USA \\ and Gary A. Hallock \\ The University of Texas at Austin \\ 1 University Station \#C0803 \\ Austin, TX 78712, USA
}

\begin{abstract}
Earlier work by Fowler, Davaney, and Hagedorn showed that the morphology of an anode streamer could be modeled as stochastic growth of a branching fractal tree in point-plane geometry. This investigation reproduces the results of that earlier study. Because one of the concerns about the earlier work is that the electric field dependence appeared to be unphysical, the model was modified to operate under assumptions that are consistent with those that have proven useful in earlier investigations. Specifically, linear electric field dependence was assumed and there is an assumed variability in the number density of available electrons. Computations using this assumption also produce the same range of morphologies that has been measured in experiments. In addition, some assessments of sensitivity to other possible variables are made. First, the sharp cutoff in the electric field strength is replaced with a presumably more realistic exponential dependence on energy. Under this assumption, it is also possible to simulate the experimentally observed behavior of anode streamers. It is shown that three possible refinements to the model have small, and likely negligible, effects. The first is using variable streamer step lengths in the calculation rather than the fixed step length used in the earlier work. The second is to assume growth at one point in the streamer makes growth in other parts somewhat less likely. The third is the assumption that the probability of a streamer making the next step in growth is influenced by the distance of the inter-electrode gap that has already been traversed.
\end{abstract}

Index Terms - Dielectric liquids, electric breakdown, trees, modeling, fractals, probability.

\section{INTRODUCTION}

MANY investigators [1-3] used high speed photography to record the shape and the growth dynamics of the prebreakdown streamers in liquids. An important result of these measurements has been that there are different modes of growth. Theoretically, these modes have been modeled using stochastic Laplacian fractal simulation with power-law (fourth-power to linear) of the field strength and threshold (cutoff) voltage $[4,5]$. This work was a logical extension of the earlier modeling of streamers in electronegative gases [6].

The theoretical approach was successful in modeling the first or the second anode mode [7] streamer as stochastic growth of a branching fractal tree in point-plane geometry. While this was a significant accomplishment, subsequent work has failed to identify broader applicability of the power law assumption for the electric field. The current work replicates and extends the results of that previous modeling approach, but uses simple assumptions that have proved to be useful in results of recent work [8] on liquid breakdown.

Two key assumptions were used in [8]. The first was that the energy, W, an electron delivered to the liquid upon collision was simply

$$
\mathrm{W}=\mathrm{eE} \lambda
$$

where $\mathrm{e}$ is the electronic charge, $\mathrm{E}$ is the local electric field, and $\lambda$ is the distance the electron travels in the field before colliding with molecules in the liquid. The second assumption [9] is that the effective electron production rate, $R(t)$, is given by

$$
R_{e}(t)=\int_{\Gamma_{c}(t)} \frac{d n_{e}}{d t} P_{0} d \Gamma,
$$


where $\mathrm{dn}_{\mathrm{e}} / \mathrm{dt}$ is the electron production rate, $\mathrm{P}_{0}$ is the recombination rate of the electrons, and $\Gamma_{\mathrm{c}}$ is the critical volume in which an electron would experience a strong enough field that it could cause ionization of the fluid upon a collision with a molecule in the field.

The approach used in this investigation was to reproduce the earlier work with power-law and threshold (cutoff) voltage. The reason for this approach was to verify that the model, and the software used to implement the model, produced results that were consistent with the previously published work. Having completed that work, the first approach to exploring alternative growth criteria is performed without the power-law assumption. Instead of power-law, it is assumed that the growth was linear in the electric field, as suggested by equation (1).

The probability of growth is assumed to be governed by equation (2), i.e., by assuming that, at any instant, not all locations in the fluid have electrons that are appropriately placed to support streamer growth. To implement this condition in the model, all of the locations that have a strong enough electric field are assigned a random number. A sharp threshold is applied to this set of random numbers to govern how growth will evolve. This random number generation is a computational approach to the replication on the variability of the electron distribution in the fluid.

The next level of refinement is to recognize that the probability of streamer growth is unlikely to be a discontinuous function of the field strength. Consequently, a probability distribution function of streamer growth was introduced in this study. The probability distribution function was established using the following conventional approach.

From equation (2), the effective electron production rate, $\mathrm{R}_{\mathrm{e}}(\mathrm{t})$, [9] can be approximated by

$$
R_{e}(t)=\int_{\Gamma_{c}(t)} \frac{d n_{e}}{d t} P_{0} d \Gamma \cong \bar{n}_{e} P_{0} \Gamma_{c}
$$

where $\bar{n}_{e}$ is the mean production rate. The incremental probability, $d P$, that an avalanche will occur between $t$ and $\mathrm{t}+\Delta \mathrm{t}$ is given by

$$
d P=(1-P) \cdot \bar{n}_{e} P_{0} \Gamma_{c}
$$

and the solution of equation (4) is given by

$$
P(t)=1-e^{-\bar{n}_{e} P_{0} \Gamma_{c} \Delta t} .
$$

Similar analysis has led to studies, by others, focusing on probabilities of streamer inception. An analytic form for the probability of breakdown inception was postulated in [10]. The analytic form for the probability distribution function is given by

$$
P(E)=1-e^{-\mu(E) \cdot S \cdot \tau},
$$

where $E$ is an electric field, $S$ is the electrode area, and $\tau$ is the pulse duration. The function of dependence on electric fields, $\mu$, is given by

$$
\mu(E)=A \cdot E^{n} .
$$

Although the functional forms are the same, it is implicitly assumed that a very different mechanism is the controlling mechanism. In the earlier work, it was assumed that streamer initiation was constrained by the likelihood of electron emission from the electrode. Here it is presumed that, at breakdown voltage levels, the electrodes and dissociative ionization, possibly photon assisted, in the bulk produce sufficient electrons. The stochastic aspect is whether or not an electron exists in a region of the fluid in which the local liquid structure provides a sufficient free path to permit streamer growth.

The work here blurs the distinction made earlier between a critical volume model which focuses the stochastic nature of growth on electron production and the field fluctuation model used by Kupershtokh and his colleagues $[10,11]$. In reality, it is expected that both the electron production and the field fluctuation, due to changes in local fluid structure, are stochastic processes. The investigation here represents the stochastic process by a random number that reflects either or both processes.

Figure 1 shows the probability of streamer growth as predicted from equation (5). Earlier work [8] suggested that critical volumes tend to be of order $10^{-9}$ to $10^{-12} \mathrm{~cm}^{3}$. In addition, studies of dc discharge onset in hexane showed that the time between pulses tended to range from about $10 \mu$ s to $1000 \mu \mathrm{s}$ [3]. Since the larger critical volumes and longer times between pulses tend to be more relevant in insulating liquids, the curves in Figure 1 focus on critical volume time products between $10^{-6}$ and $10^{-9} \mathrm{~cm}^{3} \mu \mathrm{s}$. This implies net electron production rates of about $10^{6}$ to $10^{8}$ electrons $\mathrm{cm}^{-3} \mu \mathrm{s}^{-1}$. Assuming that the production scaled linearly with density, this rate is about three orders of magnitude larger than would be expected from cosmic radiation $[12,13]$. This suggests that, if this model is correct, processes in the liquid, and not external radiation, are the dominant contributors to the electron production.

It should be recognized that in this model, the product $\Gamma_{c} \cdot \Delta t$, is a measure of the electric field strength, the size of the critical volume $\Gamma_{c}$ is a function of the applied field. Being a function of the electric field, the critical volume depends on both the geometry and the applied voltage. Similarly, $\bar{n}_{e} \cdot \mathrm{P}_{0}$ is a measure of electron density in the liquid, and thus is determined by the nature of the liquid with its various impurities. 


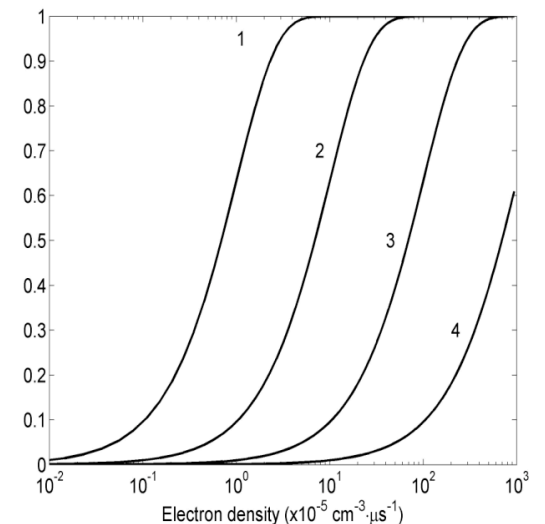

Figure 1. Probability of streamer growth. For a parametric study, the product of the critical volume and time were chosen to be $\Gamma_{\mathrm{c}} \cdot \Delta \mathrm{t}=10^{-6}, 10^{-7}, 10^{-8}$, and $10^{-9} \mathrm{~cm}^{3} \mu$ s (curves 1, 2, 3, and 4, respectively).

Using this basic set of assumptions, this paper summarizes six different investigations. The first was to replicate the assumptions and results of the earlier stochastic fractal study to validate the computational approach used in this work. The second was to achieve similar agreement with experimental observations using presumably more realistic assumptions about the key factors influencing streamer growth. The third was to replace the sharp cutoff in voltage, below which growth could not occur, with a probabilistic approach. This approach provides a more gradual transition to high probably of growth as the electric field is increased. The fourth investigated the effect of allowing the probability distribution function to vary with streamer length. The fifth examines the effect of weighting the likelihood of a growth step occurring by the relative local field strength at the location of the step. Finally, the sixth examined the effect of permitting step lengths for streamer growth to be longer than to the nearest grid point, as was assumed in the earlier work.

\section{SOFTWARE VALIDATION}

Since not all of the software used in the original investigation was available for this work, some new coding was required. The model simulated the growth of high-speed filamentary streamers during high-voltage breakdown in liquid dielectrics [14]. The computational approach was to compute the stochastic growth of Laplacian fractals on a 3-dimensional Cartesian grid. In the model, after examining neighbor sites one grid-step away from the streamer, where voltage difference between the site and the tree exceeds a threshold level, the approach is to compare against a random number, using the power-law, to determine if growth occurs.

Figure 2 shows the results that replicate the earlier investigation $[4,5]$. Specifically, the assumption of a fourth or third power dependence on the electric field results in sparse structures. Quadratic laws yield sparse structures with the threshold (cutoff) voltage of 0.05 and 0.07 . The linear cases with the threshold of $0.05,0.15$, and 0.2 are the densest. These data also show, that assuming a larger difference between the voltage in the streamer and at the grid point toward which it may grow, i.e., assuming a higher threshold, yields sparser structures.

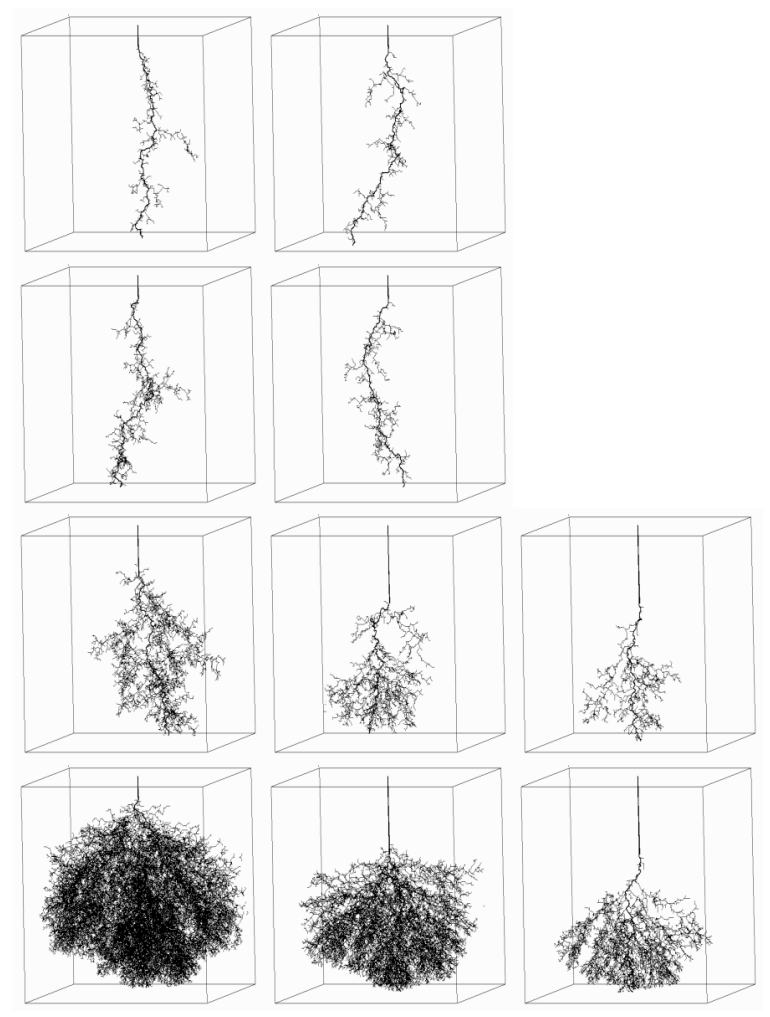

Figure 2. Fourth-law examples with the threshold of 0.05 and 0.07 in the first row, third-law examples with 0.05 and 0.07 in the second row, square-law examples with $0.05,0.15$, and 0.2 in the third row, and linear-law examples with $0.05,0.12$, and 0.2 in the last row.

\section{STOCHASTIC GROWTH WITH A SHARP VOLTAGE CUTOFF}

The first approach to alternative growth criteria was to maintain the concept of a sharp voltage cutoff, but to explore theoretically the regime in which the likelihood of having an electron capable of contributing to streamer growth was a variable. This assumption was implemented by assigning a random number to each site and assuming that no growth can occur if the random number is below an arbitrary threshold. This is a reasonable assumption for streamer growth in an insulating liquid for voltages slightly above the inception voltage.

Figure 3 shows the simulation results with the assumed threshold for the random numbers. The bushiest result, in the upper left of the figure, is obtained with the lowest field and the highest electron density. As the electric field strength is increased or the electron density is decreased, the growth pattern becomes increasingly sparse. This reproduces the data in Figure 2 without having to assume the differing structure of the growth is determined by a dependence on electric fields to different powers. 


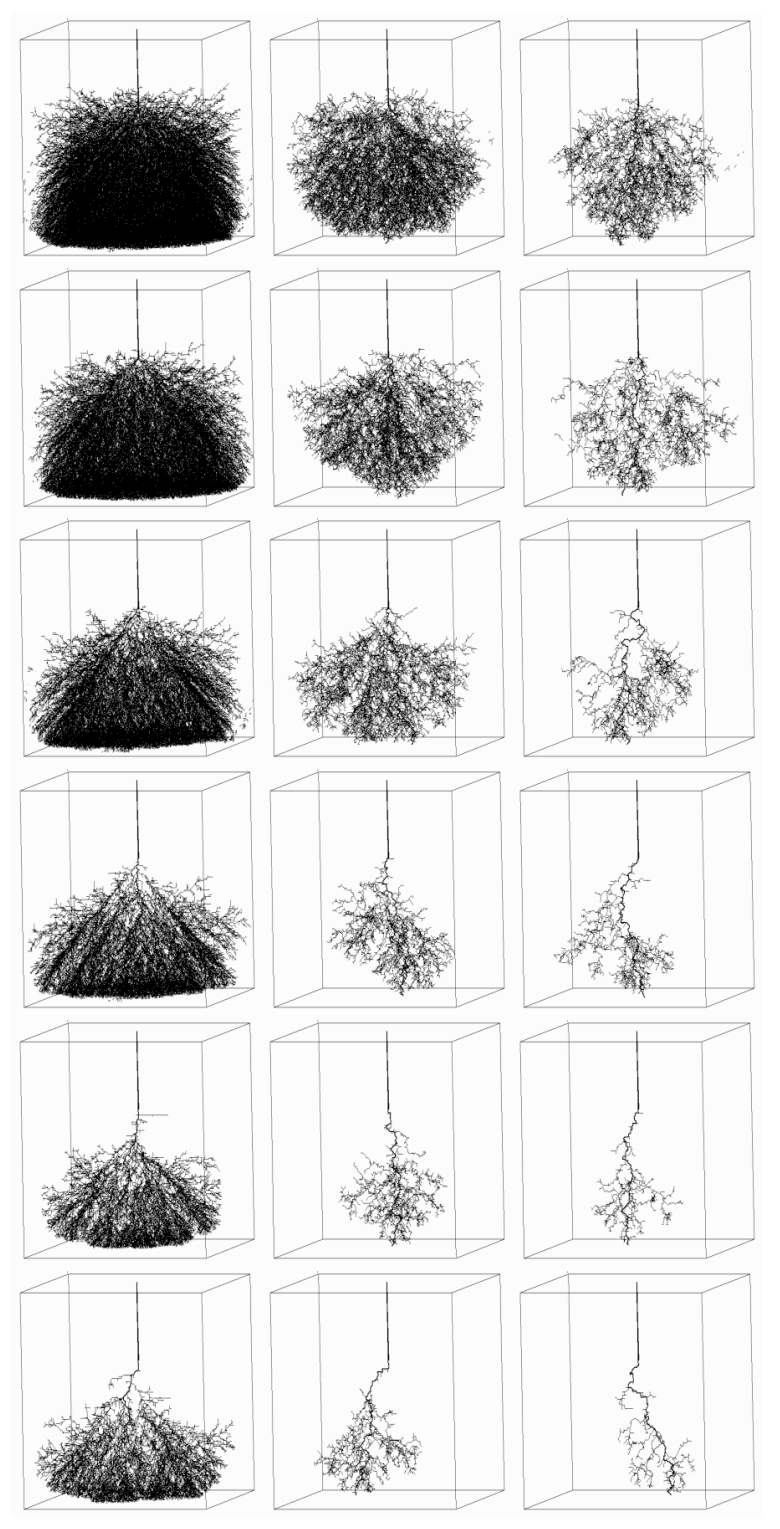

Figure 3. Stochastic growth of the fractal tree without power-law. Threshold voltage is $1,1.25,1.5,1.75,2.0$, and 2.1 from top to bottom, respectively. Threshold of random number is $0.5,0.1$, and 0.02 from left to right, respectively.

\section{STOCHASTIC GROWTH WITH A PROBABILITY DISTRIBUTION FUNCTION}

Figure 4 shows the growth of the fractal tree using the probability distribution function shown in Figure 1. Various shapes of calculated streamers are shown as a function of the product of the size of the critical volume and time, $\Gamma \cdot \Delta \mathrm{t}$. The stochastic growth with $\Gamma \cdot \Delta \mathrm{t}$ between 1 and 0.2 is bushy, and the growth is sparse at $\Gamma \cdot \Delta t=0.02$. The probability distribution function, where $\Gamma \cdot \Delta \mathrm{t}$ is less than 0.01 , produced very similar results. Under $0.01, \Gamma \cdot \Delta t$ does not affect the shape of the fractal tree, but affects only the stochastic time tick. These results are expected, as they show that reducing either the size of the critical volume or time results in sparse structures.

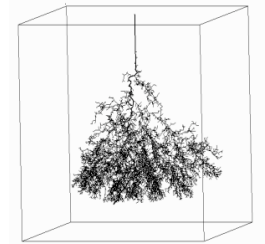

$\Gamma_{\mathrm{c}} \cdot \Delta t=0.5$

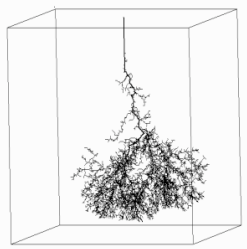

0.2

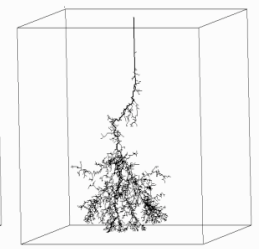

0.1

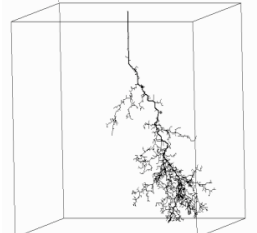

$\Gamma_{\mathrm{c}} \cdot \Delta t=0.05$

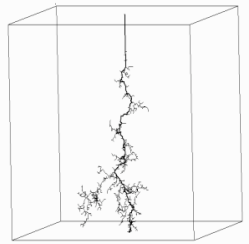

0.02
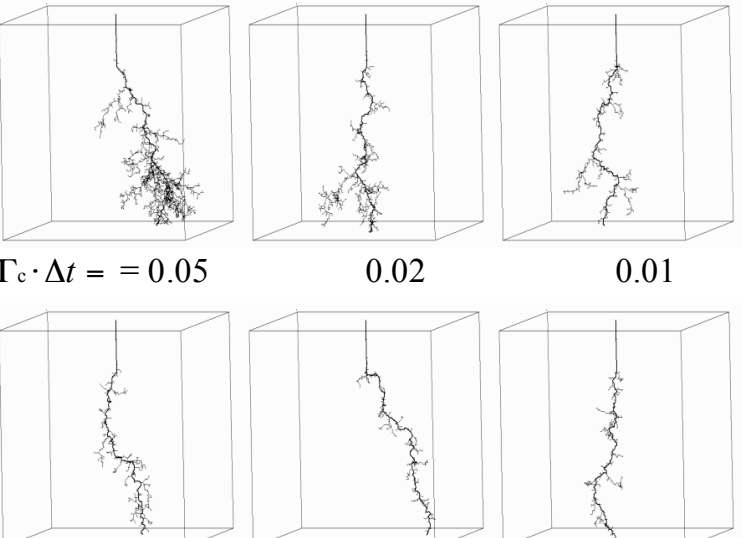

$\Gamma_{\mathrm{c}} \cdot \Delta t=0.005$

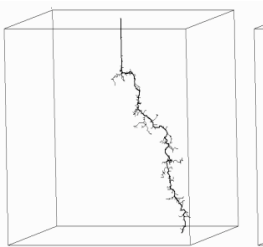

0.002

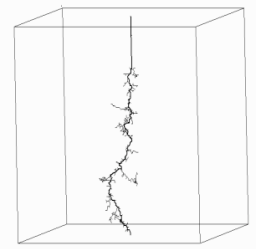

0.001

Figure 4. Simulations of stochastic growth with probability distribution function.

\section{PROBABILITY DISTRIBUTION AS A FUNCTION OF DISTANCE}

In the previous section, the probability distribution function was presumed to be independent of previous growth. This is consistent with assuming that the electron density in the fluid toward which the streamer is propagating is determined primarily by fluid properties, and that the electric field of interest is that very near (i.e., within one or, at most, a few micrometers in front of) the streamer tips. This field does not change significantly as the streamer propagates. While these assumptions appear reasonable, it is prudent to make at least some assessment of the sensitivity of the results to the assumption.

In particular, an unanswered question is whether the electric field well ahead of the streamer influences the availability of effective electrons. The previous assumption is that nothing that happens in a volume of liquid influences the streamer propagation until that volume is included in a critical volume.

The form of the probability distribution function in equation (5) is used for this investigation. In this analysis, it is assumed that the positional variation is given by:

$$
\bar{n}_{e} \mathrm{P}_{0} \Gamma_{\mathrm{c}} \cdot \Delta t=\kappa 10^{\mathrm{x}}, \quad x=\frac{\log \frac{a}{b}}{c}
$$

where $a$ and $b$ are distances shown in Figure $5, \kappa$ is an appropriate constant to yield the previous probability distribution function at $\mathrm{x}=0$, and $\mathrm{c}$ is a scaling constant. If $\mathrm{c}=$ $\log 2$, it means that the probability of initiating the next step in streamer growth is changing gradually to meet the value 0.1 when the distance ratio, $a / b$, is $1 / 2$, and the value 0.01 when $\mathrm{a} / \mathrm{b}$ is $1 / 4$. 
The effects of assuming a probability distribution function that is the presumed function of distance are shown in Figure 6 . The result with $\mathrm{c}=\log 2$ shows similar shape of the second mode. While there are small changes, the changes do not affect the streamer growth rate nor do they appear to be consistent with experimental data. So, it appears that a probability distribution function that is independent of the degree of previous streamer growth may be a reasonable assumption.

Obviously, the examination of a single possible alternative does not constitute proof of such independence. Since there are no credible models of a positional dependence, no further analysis was done.

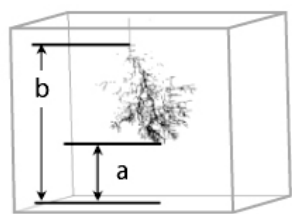

Figure 5. Parameter definition.

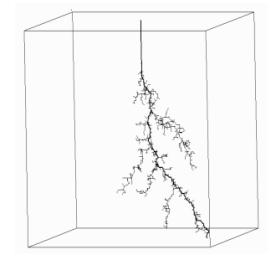

$\mathrm{c}=\log 2$
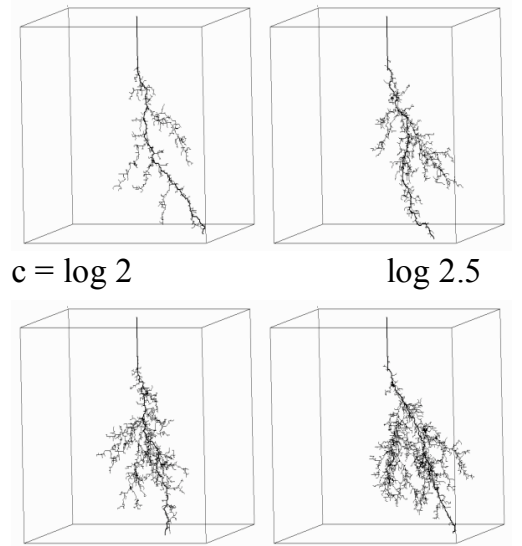

$\mathrm{c}=\log 3$

$\log 2.5$

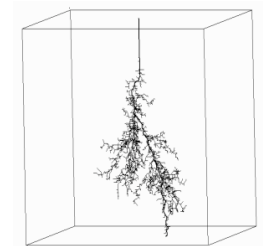

$\mathrm{c}=\log 4$

Figure 6. Stochastic growth with probability distribution function as a function of the gap distance.

\section{Relative Weighting Factor}

The probability distribution function of each candidate grid point in this study is defined from the electric field of each site, and the fields of the other sites do not affect each other in this decision process of streamer connection. There may be a possible influence, however. It is plausible to presume that sites having relatively higher fields have higher possibility of a streamer connection, and those having relatively lower fields have lower possibility. To incorporate this concept into the model, a relative weighting factor, $\alpha$, is assumed. The relative weighting factor is defined as

$$
\alpha=\varphi / \varphi_{\text {avg }}
$$

where $\varphi$ is the electric field of each available site and $\varphi_{\text {avg. }}$ is the average $\varphi$ of all the available sites. In this investigation, an available site was a grid point that was one grid step from the streamer of the electrode.

The process used to assess the influence of $\alpha$ on the streamer growth is first to assign a constant probability, $\mathrm{k}$, to each candidate site. Then, random numbers are generated for candidates. If $\alpha \cdot \mathrm{k}$ is greater than the random number, then the site is connected to the streamer. Therefore, $\alpha$ is the determining factor for growth.

Figure 7 shows the results with $\alpha$ being determined from the code using equation (8) and $\alpha$ ranging from 0.01 to 0.0002 . Streamers at the top electrode are shown in all cases because no threshold electric field was assumed, so every site can initiate a streamer. As expected, the streamer becomes sparser as the probability of growth becomes smaller.

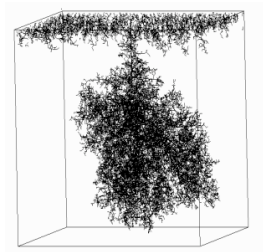

$\mathrm{k}=0.01$

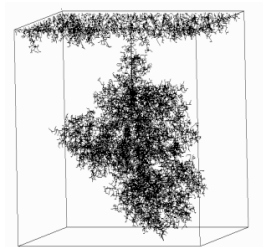

$\mathrm{k}=0.002$

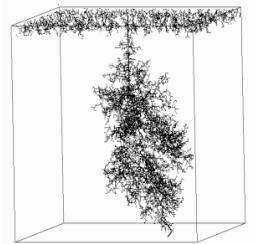

$\mathrm{k}=0.0005$

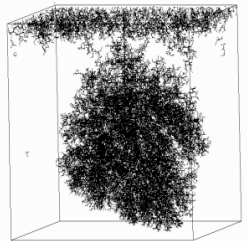

0.005

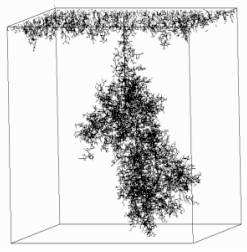

0.001

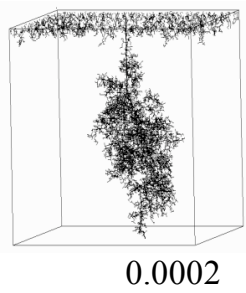

Figure 7. Stochastic growth with a weighting factor.

To achieve better correlation with experimental observations, the sharp threshold, $\mathrm{k}$, is replaced by the probability distribution function assumed in equation (5). The probability for each site is obtained from that function according to the electric field. The probability is multiplied by the scaling factor $\alpha$. If the product is greater than a random number, then the candidate becomes a part of the streamer.

The result of stochastic growth combining the weighting factor $\alpha$ and the probability distribution function is shown in Figure 8. An interesting aspect to this growth model is that the growth is sparse at the initiating electrode and becomes bushier with time. This behavior has been observed in initiation studies using spherical electrodes [15] and in some higher resolution studies of initiation from a point electrode [16]. 

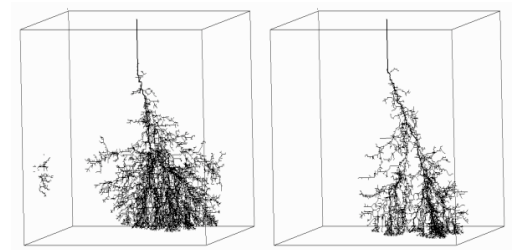

$\Gamma_{c} \cdot \Delta t=0.05$

0.02

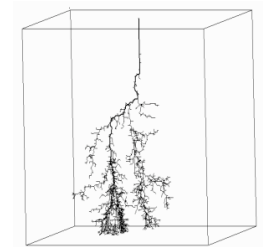

$\Gamma_{c} \cdot \Delta t=0.01$

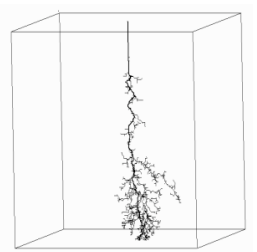

0.005

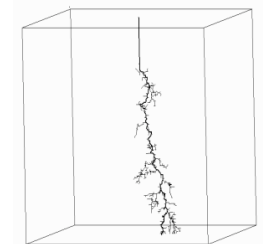

$\Gamma_{c} \cdot \Delta t=0.002$

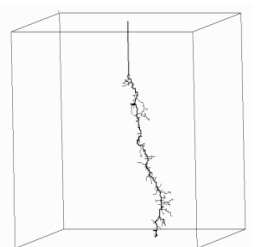

0.001

Figure 8. Stochastic growth with the onset determined using both a probability distribution function and a weighting factor that permits greater growth probability in areas of higher field.

\section{STOCHASTIC GROWTH WITH RANDOM LENGTH OF STREAMER}

In the model used, the streamer growth step was limited to the nearest neighbor on the grid. Given small scale density fluctuations in liquids, it is possible that different growth step lengths could occur. Because of this possibility, random length streamers were examined.

The process used to determine streamer length is to calculate $\varphi_{\text {avg }}$, an average electric field of neighbor sites of one grid-step away from the streamer. If the electric field of a site is greater than or equal to $\varphi_{\text {avg }}$, then the site is a candidate for growth of two or three grid positions. If the field is less than $\varphi_{\text {avg }}$, then the site is a candidate for one or two grid positions. The actual growth is selected by generating a random number for each site and comparing it to 0.5 . If the random number is greater than or equal to 0.5 , then the streamer step length at that site is a longer one, two or three. If the random number is less than 0.5 , then the length at that site is a shorter one, one or two.

Figure 9 shows the results from the application of this algorithm. Overall, the streamers are more directional toward the cathode, but the main trend is very close to Figure 4. It is not clear whether this assumed variation provides a better or worse representation of the experimental data.

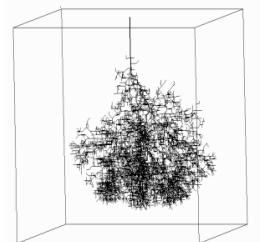

$\Gamma_{c} \cdot \Delta t=1$

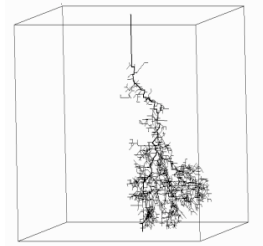

$\Gamma_{c} \cdot \Delta t=0.1$

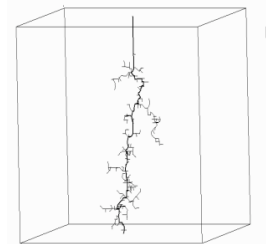

$\Gamma_{c} \cdot \Delta t=0.01$

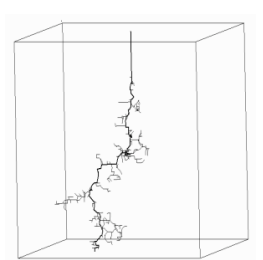

$\Gamma_{c} \cdot \Delta t=0.001$

Figure 9. Simulations of the random length of streamer with probability distribution function.

\section{DISCUSSION}

The work of Fowler, Devaney, and Hagedorn showed a path for modeling the morphology of prebreakdown streamers in liquids. Although they were successful in replicating experimentally observed patterns, the success underscored the fact that morphology was probably not uniquely related to the assumed underlying physical processes. Specifically, they achieved the experimentally observed change in streamer morphology by assuming the growth was proportional to $\mathrm{E}^{\mathrm{n}}$, where $n$ was an integer between 1 and 4. As $n$ is varied, the calculated shape changed, being the most bushy at $n=1$ and the most sparse at $n=4$. There has been no investigation that showed that the variable power dependence was manifested in any other way.

Consequently, this study investigated the possibility of replicating their results using a simple description of the underlying processes that had proved successful in earlier studies of the breakdown process [8]. The key assumptions of that model is that there is a critical volume in the liquid in which the electric field is strong enough to sustain streamer growth, and that the production, in the volume of electrons with a sufficiently long free path is a stochastic process. It has been shown that replacing the $\mathrm{E}^{\mathrm{n}}$ assumption in the model developed by Fowler, Devaney, and Hagedorn with these 
assumptions also yields the experimentally observed morphology. Moreover, the predicted behavior is that as the electric field is reduced and/or the electron density is increased, the streamers become bushier. This is consistent with experimental observations in streamers from point cathodes [17]. This correlation suggests that the model may be equally valid for anode and cathode streamers. That is not a surprising observation since there is nothing in the model that is polarity dependant.

The robustness of the correlations was explored by recalculating behavior with a probabilistic, rather than abrupt, onset of breakdown. This approach is consistent with the other work on electrical breakdown and reduces the arbitrary assumptions that must be made in the model. Investigations using a position dependant probabilistic model, a fielddependant probabilistic model, and a model that permitted variation in the length of growth steps showed that these assumptions make only small differences in the calculated results. These differences are likely too small to be resolved experimentally given the precision of today's experiments.

\section{CONCLUSIONS}

This investigation showed that a simple model of the breakdown process coupled with a computational approach based on the stochastic growth of Laplacian fractals on a 3dimensional cartesean grid can produce streamer morphologies over the range detected experimentally. While the model appears to fit the data with plausible values for electron production and time, neither the data not the models are precise enough to claim an unambiguous proof of consistency. Rather, the work shows that the simulation approach, the available data, and the presumed breakdown model can yield a consistent description of prebreakdown streamers in liquids.

\section{ACKNOWLEDGEMENTS}

The authors wish to thank J. E. Devaney for providing copies of most of the computer programs used in the earlier work. This investigation was supported by the Office of Naval Research.

\section{REFERENCES}

[1] S. Otsuki, K. Yamazawa, K. Sugiura, and H. Yamashita, "A study of three-dimensional measurement of pre-breakdown streamer in dielectric liquids," IEEE Conf. on Electr. Insul.and Dielectr. Phenom., Vol. 2, pp. 644-647, 1997

[2] P. Rain and O. Lesaint, "Prebreakdown phenomena in mineral oil under step and ac voltage in large-gap divergent fields," IEEE Trans. Dielectr. Electr. Insul., Vol. 1, pp. 692-701, 1994.

[3] E. Kelley, et al., "Measurement of partial discharges in Hexane under dc voltage," IEEE Trans. Electr. Insul., Vol. 24, pp.1109-1119, 1989.

[4] H. A. Fowler, J. E. Devaney, and J. G. Hagedorn, "Shaping of filamentary streamers by the ambient field," IEEE Conf. on Electr. Insul. and Dielectr. Phenom., pp. 132-136, 1999.

[5] H. A. Fowler, J. E. Devaney, and J. G. Hagedorn, "Growth model for filamentary streamers in an ambient field," IEEE Trans. on Dielectr. Electr. Insul., Vol. 10, pp. 73-79, 2003.

[6] L. Niemeyer, L. Ullrich, and N. Wiegart, "The mechanism of leader breakdown in electronegative gases," IEEE Trans. Electr. Insul., Vol. 24, pp. 309-324, 1989.

[7] R. E. Hebner, "Measurement of electrical breakdown in liquids," Proc. of the NATO Advanced Study Institute on the Liquid State and its
Electrical Properties, Sintra, Portugal, Plenum Pub. Corp., New York City, NY, pp. 519-537, 1987.

[8] M. Kim and R. E. Hebner, "Initiation from a point anode in a dielectric liquid," IEEE Trans. Dielectr. Electr. Insul., Vol. 13, pp. 1254-1260, 2006.

[9] S. K. Venkatesh and M. S. Naidu, "Experimental and theoretical investigations on the statistics of time lags to corona inception and breakdown of $\mathrm{SF}_{6}$ in non-uniform electric fields," Gaseous Dielectrics, Vol. VIII, Christophorou and Olthoff, Eds. New York: Plenum, 1998, pp. 115-160.

[10] A. L. Kupershtokh and D. I. Karpov, "Stochastic features of initiation of liquid dielectric breakdown at small area of positive electrode," IEEE $13^{\text {th }}$ Int. Conf. on Dielectr. Liquids, pp. 203-206, 1999.

[11] A. P. Ershov and A. L. Kupershtokh, "Fluctuation model of liquid dielectrics breakdown with incomplete charge relaxation," IEEE $11^{\text {th }}$ Int. Conf. on Dielectr. Liquids, pp. 194-198, 1993.

[12] S. A. Boggs, "Sulphur Hexafluoride-A complex dielectric," Electri. Insul. Mag., Vol. 5, pp. 16-21, 1989.

[13] W. F. Schmidt, M. Kim, and R. E. Hebner, "Initiation from a point anode in a dielectric liquid," IEEE Trans. Dielectr. Electr. Insul., Vol. 14, pp. 762-763, 2007.

[14] H. A. Fowler, J. E. Devaney, and J. G. Hagedorn, "User's guide to CADMUS, a simplified parallel code for Laplacian-fractal growth," NISTIR 6180, June 1998.

[15] E. F. Kelly and R. E. Hebner, "Prebreakdown phenomena between sphere-sphere electrodes in transformer oil," Appl. Phys. Lett., Vol. 38, pp. 231-233, 1981.

[16] G. J. Fitzpatrick, E. O. Forster, R. E. Hebner, and E.F. Kelley, "Prebreakdown cathode processes in liquid hydrocarbons," IEEE Trans. on Electr. Insul., Vol. EI-22, pp. 453-458, 1987.

[17] R. E. Hebner, "Factors contributing to streamer morphology," Proc. of 2002 IEEE 14th International Conference on Dielectr. Liquids, pp. 155$158,2002$.

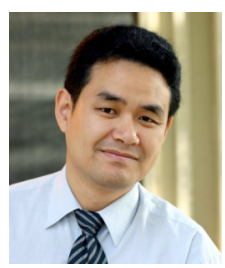

Minkyu Kim was born in Seoul, Korea in 1971. He received his B.S. degree from Chung-Ang University, Seoul, Korea, in 1994 and his M.S. degree from Seoul National University, Seoul, Korea, in 1997, both in electrical engineering. He is currently pursuing his $\mathrm{Ph} . \mathrm{D}$. degree in electrical engineering at the University of Texas, Austin, TX.

Previously, he was with Samsung Electronics from 1997 to 2002 , and worked in the areas of processing plasmas, plasma physics, and control systems. His research interests include streamer analysis, processing plasmas, plasma physics, and electromagnetic waves from plasmas.

Robert E. Hebner, Ph.D., (S'70-M'71-SM'83-F'93) is Director of the Center

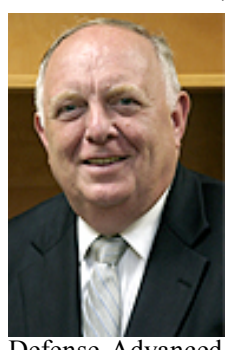
for Electromechanics at the University of Texas at Austin. The Center develops technology, primarily novel motors, generators, and suspension components, and teams with companies to get the technology into the market.

Previously, Dr. Hebner was the acting Director of the U.S. National Institute of Standards and Technology (NIST). In addition, he has directed NIST's Electronic and Electrical Engineering Laboratory, a laboratory with a staff of more than 250 . He also worked at the Defense Advanced Research Projects Agency where he developed programs to improve semiconductor manufacturing.

Throughout his career, Dr. Hebner has been active in having authored or coauthored more than one hundred technical papers and reports. He has extensive experience in international technology programs. This work included the modernization of the measurement systems needed to support global trade and the assessment of the effectiveness of government technology programs in stimulating domestic economies. 


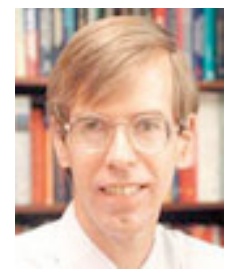

Gary A. Hollock, Ph.D., (S'76-M'80) received his B.S., M.S., and Ph.D. degrees in electrical engineering from Rensselaer Polytechnic Institute, Troy, NY. He joined the Department of Electrical and Computer Engineering at the University of Texas at Austin in 1984, after working for several years at Oak Ridge National Laboratory in Oak Ridge, Tennessee. Dr. Hallock has a number of teaching interests at both the undergraduate and graduate level. He has received a number of honors and awards, including the Dumont Award, EFAC Faculty Excellence Award, NASA Certificate of Appreciation, and Dads' Association Centennial Teaching Fellowship.

Dr. Hallock's research is in plasma science and involves two different areas in this applied science field. The first involves high performance Hall-type plasma thrusters, which are being developed for stationkeeping of the next generation of communications satellites. Dr. Hallock and his students are concerned with the basic plasma aspects of the thrusters and the effects of the emitted plasma plume on the satellite microwave communication signals. The second area of focus involves the development of sophisticated diagnostics to be used in studies of plasma confinement and turbulence in controlled fusion experiments. Examples of these diagnostics include far-infrared interferometers and phase contrast imaging systems for studying plasma turbulence. 